\title{
Advanced Wave-Equation Migration
}

\author{
Lianjie Huang and Michael C. Fehler \\ Los Alamos Seismic Research Center, Los Alamos National Laboratory, Los Alamos, NM 87545, USA
}

\begin{abstract}
Wave-equation migration methods can more accurately account for complex wave phenomena than ray-tracing-based Kirchhoff methods that are based on the high-frequency asymptotic approximation of waves. With steadily increasing speed of massively parallel computers, wave-equation migration methods are becoming more and more feasible and attractive for imaging complex $3 \mathrm{D}$ structures. We present an overview of several efficient and accurate wave-equation-based migration methods that we have recently developed. The methods are implemented in the frequency-space and frequency-wavenumber domains and hence they are called dualdomain methods. In the methods, we make use of different approximate solutions of the scalar-wave equation in heterogeneous media to recursively downward continue wavefields. The approximations used within each extrapolation interval include the Born, quasi-Born, and Rytov approximations. In one of our dual-domain methods, we use an optimized expansion of the square-root operator in the one-way wave equation to minimize the phase error for a given model. This leads to a globally optimized Fourier finitedifference method that is a hybrid split-step Fourier and finitedifference scheme. Migration examples demonstrate that our dualdomain migration methods provide more accurate images than those obtained using the split-step Fourier scheme. The Bornbased, quasi-Born-based, and Rytov-based methods are suitable for imaging complex structures whose lateral variations are moderate, such as the Marmousi model. For this model, the compulational cost of the Born-based method is almost the same as the split-step Fourier scheme, while other methods takes approximately 15-50 per cent more computational time. The globally optimized Fourier finite-difference method significantly improves the accuracy of the split-step Fourier method for imaging structures having strong lateral velocity variations, such as the SEG/EAGE salt model, at an approximately 30 per cent greater computational cost than the split-step Fourier method.
\end{abstract}

\section{INTRODUCTION}

Since the inception of the Kirchhoff migration method in late $1970 \mathrm{~s}$ (Schneider, 1978), the ray-based migration method has been widely used in industry because of its remarkable high efficiency. Rays are high-frequency asymptotic approximation of waves, therefore, it is difficult for a ray migration scheme to accurately image complex structures where wave phenomena are important. Many improvements have been made to make the ray migration more reliable and different ray migration methods have been developed during last two decades (e.g. Wiggins, 1984; Keho and Beydoun, 1988; Zhu, 1988; Geoltrain and Brac, 1993; Gray and May, 1994; Moser, 1994; Fei et al., 1996; Bevc, 1997; Audebert et al., 1997; Hanitzsch, 1997; Hildebrand and Fehler, 1998; Tygel et al., 1998; Abma et al., 1999; Dellinger et al., 2000; Hokstad, 2000; Romero et al., 2000;
Sun et al., 2000). To date, the ray-based migration method is still the most commonly used imaging tool in industry.

Wave propagation in continuous and heterogeneous media is governed by the wave equation. This partial differential waveequation is often numerically solved using different methods to simulate complex wave phenomena. Migration imaging is a backpropagation of wavefields recorded on the surface of the Earth. Imaging a complex 3D structure requires a great number of back propagation steps, and therefore, it needs an accurate and efficient imaging scheme. To achieve a high efficiency and reduce the computer memory requirement when using wave equation for migration, Claerbout pioneered research in this area by using the one-way wave equation and introducing different approximations to the square-root operator in the equation in 1970s. He summarized wave-equation migration methods developed by himself and his colleagues in a book published in 1985 (Claerbout, 1985). In one-way wave-equation-based methods, wavefields recorded on the surface of the Earth are downward continued into the Earth and an imaging condition is applied at each position within the Earth to obtain the image of the Earth's interior. Most of the methods introduced in Claerbout's 1985 book make use of finite-difference schemes to solve the approximations of the square-root operator in the one-way wave equation. Haje (1991) introduced a finitedifference migration method using McClellan transformations.

Some wave-equation migration methods are based on use of the Fourier transform and are carried out in the frequency-space and frequency-wavenumber domains. We refer them to as the dualdomain migration methods. The Fourier transform-based methods exactly implement the transverse Laplacian operators in the wave equation, and consequently, they minimize the artificial numerical dispersion effects that commonly occur in the finite-differencebased methods. Gazdag (1978) developed the phase-shift migration method and Stolt (1978) proposed the F-K migration scheme. Both methods require a homogeneous velocity model. To handle lateral velocity variations, Gazdag and Sguazzero (1984) introduced the phase-shift plus interpolation (PSPI) method, and Stoffa et al. (1990) proposed the split-step Fourier (SSF) approach. Wu and Jin (1997) and Jin and Wu (1999) introduced a windowed Fourier transform-based method. Le Rousseau and De Hoop (1999) and de Hoop et al. (2000) developed a high-order Fourier transformbased method that obtains increased accuracy at a cost of increasing the number of Fourier transforms used. Ristow and Rühl (1994) developed a hybrid split-step Fourier and finite-difference approach called the Fourier finite-difference method. Xie and Wu (1998) introduced an alternative hybrid split-step Fourier and finitedifference scheme.

In recent years, we have developed a suite of wave-equation migration methods that are based on one-way wave propagation and implemented in the frequency-space and frequency-wavenumber domains (Huang and Wu, 1996a, b; Feller and Huang, 1998; Huang et al., 1998, 1999a, b; Huang and Fehler, 2000a, b, 2001). 
We have also used some of these methods for modeling primary forward wave propagation in random media (Fehler and Huang, 1998; Huang and Fehler, 1998b; Fehler et al., 2000). In this paper, we present an overview of these dual-domain migration methods. In the methods, we applied the Born, quasi-Born, and Rytov approximations within each extrapolation interval during wavefield downward continuation. In one of the methods, we optimized the Fourier finite-difference method for the entire model. We give a brief description of the methods and present migration images obtained using synthetic datasets for the Marmousi model and the SEG/EAGE salt model. The images obtained using our dual-domain methods are more accurate and reliable than those obtained using the the split-step Fourier scheme (Stoffa et al., 1990; Huang and Fehler, 1998a), which is a small-angle approximation of the Rytov-based method.

Compared to ray migration methods, the advantage of waveequation migration methods is that they can more accurately account for complex wave phenomena, while their disadvantage is that they are generally more computationally intensive than ray migration methods. However, with steadily increasing speed of massively parallel computers, the wave-equation migration schemes are now becoming more and more feasible and attractive for imaging complex 3D structures.

\section{WAVEFIELD EXTRAPOLATION EQUATIONS}

We give the wavefield extrapolation equations for our dual-domain wave-equation migration methods including the extended local Born Fourier (ELBF), quasi-Born Fourier (QBF), extended 10cal Rytor Fourier (ELRF), and globally optimized Fourier finitedifference (GOFFD) approaches. The ELBF, QBF, and ELRF methods are derived using the two-way wave equation and a one way Green's function, while the GOFFD method is based on the one-way wave equation.

\section{ELBF Method}

In the ELBF method (Huang et al., 1999b), the Born approximation is applied within each extrapolation interval. To extrapolate the wavefield from depth level at $z_{i}$ to another level at $z_{i+1}=z_{i}+\Delta z$ where $\Delta z$ is the extrapolation interval, the incident and scattered wavefields at $z_{i+1}$ are extrapolated using the known wavefield $P\left(x, z_{i} ; \omega\right)$ to form the wavefield at $z_{i+1}$ given by

$$
P\left(x, z_{i+1} ; \omega\right)=P_{0}\left(x, z_{i+1} ; \omega\right)+P_{s}^{B}\left(x, z_{i+1} ; \omega\right),
$$

where the incident wavefield $P_{0}\left(x, z_{i+1} ; \omega\right)$ is obtained by

$$
P_{0}\left(x ; z_{i+1} ; \omega\right)=\mathcal{F}_{k_{x}}^{-1}\left\{e^{i k_{z}\left(z_{i}\right) \Delta z} \mathcal{F}_{x}\left\{P\left(x_{1} z_{i} ; \omega\right)\right\}\right\}
$$

with the vertical wavenumber $k_{z}$ given by

$$
k_{z}\left(z_{i}\right)=\sqrt{\frac{\omega^{2}}{v_{0}^{2}\left(z_{i}\right)}-k_{x}^{2}}
$$

In eqs. (1)-(3), $\omega$ is the circular frequency. In eq. (2), $\mathcal{F}_{x}$ represents the Fourier transform over $x$ and $\mathcal{F}_{k_{x}}{ }^{1}$ represents the inverse Fourier transform over $k_{x}$. In eq. (3), $v_{0}\left(z_{i}\right)$ represents a reference velocity of the medium within the interval from $z_{i}$ and $z_{i+1}$, which is generally chosen as the reciprocal of the average slowness. The Born scattered wavefield $P_{s}^{B}\left(x, z_{i+1} ; w\right)$ is given by

$$
\begin{aligned}
P_{s}^{B}\left(x, z_{i+1} ; \omega\right) \approx & \mathcal{F}_{k_{x}}^{-1}\left\{\sigma\left(z_{i}\right) e^{i k_{z}\left(z_{i}\right) \Delta z}\right. \\
& \left.\times \mathcal{F}_{x}\left\{\left[i \omega \Delta s\left(x, z_{i}\right) \Delta z\right] P\left(x, z_{i} ; \omega\right)\right\}\right\}
\end{aligned}
$$

where $\Delta s$ is the slowness perturbation and the parameter $\sigma$ is given by

$$
\sigma \equiv \frac{k_{0}}{k_{z}}=1+A
$$

with the term $A$ approximated by

$$
\begin{aligned}
A \approx & 0.5\left(\frac{k_{x}}{k_{0}\left(z_{i}\right)}\right)^{2}+0.375\left(\frac{k_{x}}{k_{0}\left(z_{i}\right)}\right)^{4} \\
& +0.3125\left(\frac{k_{x}}{k_{0}\left(z_{i}\right)}\right)^{6}+0.2734375\left(\frac{k_{x}}{k_{0}\left(z_{i}\right)}\right)^{8}
\end{aligned}
$$

\section{QBF Method}

In the quasi-Born Fourier method (Huang and Fehler, 2000b), the scattered wavefield in eq. (1) is replaced by the quasi-Born scattered wavefield given by

$$
P_{s}^{q B}\left(x, z_{i+1} ; \omega\right) \approx \frac{P_{0}\left(x, z_{i+1} ; \omega\right) P_{s}^{B}\left(x, z_{i+1} ; \omega\right)}{P_{0}\left(x, z_{i+1} ; \omega\right)-P_{s}^{B}\left(x, z_{1+1} ; \omega\right)}
$$

\section{ELRF Method}

The ELRF method (Huang et al., 1999a) makes use of the wavefield extrapolation equation given by

$$
P\left(x, z_{i+1} ; \omega\right) \approx P_{0}\left(x, z_{3+1} ; \omega\right) e^{\phi_{s}\left(x, z_{i}+1 ; \omega\right)}
$$

where the complex exponential term $\phi_{s}\left(x, z_{i+1} ; w\right)$ is given by

$$
\begin{gathered}
\phi_{s}\left(x, z_{i}+\Delta z ; \omega\right)=\frac{P_{s}^{B}\left(x, z_{i+1} ; \omega\right)}{P_{0}\left(x, z_{i+1} ; \omega\right)} \\
\approx \frac{P_{s}^{B}\left(x, z_{i+1} ; \omega\right) P_{0}^{*}\left(x, z_{i+1} ; \omega\right)}{P_{0}\left(x, z_{i+1} ; \omega\right) P_{0}^{*}\left(x, z_{i+1} ; \omega\right)+\delta}
\end{gathered}
$$

where the superscript " $*$ " denotes the complex conjugate and $\delta$ is a small real number which can be chosen as

$$
\delta=\zeta\left[\max \left\{P_{0}\left(x_{1} z_{i+1} ; \omega\right) P_{0}^{*}\left(x, z_{i+1} ; \omega\right)\right\}\right]
$$

with a small real number of $\zeta$. In eq. $(10)$, the maximum value is searched for all lateral positions at $z_{1+1}$ for a given $\omega$. In practice, the value of $\zeta$ can be 0.01 to 0.05 . We showed that the split-step Fourier method is a small-angle approximation of the extended local Rytov Fourier method (Huang et al., 1999a).

\section{GOFFD Method}

The GOFFD method (Huang and Fehler, 2000a) is based on the one-way wave equation in the frequency-space domain given by

$$
\frac{\partial P(x, z ; \omega)}{\partial z}=i Q(x, z ; \omega) P(x, z ; \omega)
$$


with operator $Q$ defined as

$$
Q \equiv \sqrt{\frac{\omega^{2}}{v^{2}(x, z)}+\frac{\partial^{2}}{\partial x^{2}}}
$$

where $v$ is velocity. Making use of a reference velocity $z$, operator $Q$ is approximated by

$$
Q \approx \sqrt{k_{0}^{2}+\frac{\partial^{2}}{\partial x^{2}}}+k_{0}\left(\frac{1}{m}-1\right)-k_{0} \frac{a(m-1) X_{0}^{2}}{1-b\left(1+m^{2}\right) X_{0}^{2}}
$$

where $a$ and $b$ are chosen by an optimization scheme to minimized the phase error for a given model, and

$$
\begin{aligned}
m & \equiv \frac{v}{v_{0}}, \\
k_{0} & \equiv \frac{\omega}{v_{0}} \\
X_{0}^{2} & \equiv-\frac{1}{k_{0}^{2}} \frac{\partial^{2}}{\partial x^{2}},
\end{aligned}
$$

where $v_{0}$ is a reference velocity that must be the minimum value of velocities at all lateral positions within an extrapolation interval (Ristow and Rühl, 1994; Huang and Fehler, 2000a). Extrapolation of wavefields using eq. (11) and the combination of the first two tems on the right side of eq. (13) results in the split-step Fouricr propagator. The corresponding operator of the third term in eq. (13) is a compensation operator for the split-step Fourier prop. agator to improve its accuracy when lateral velocity variations are strong. This operator can be implemented using an implicit finitedifference method (Claerbout, 1985). Consequently, the wavefieldextrapolation method using eqs. (11) and (13) is a hybrid approach. When $m=3, a=0.47826, b=0.43848$.

\section{MIGRATION EXAMPLES}

We use the dual-domain wave-equation migration methods described in the previous section to migrate synthetic datasets from two standard models, the Marmousi model and the SEG/EAGE salt model. The images are compared with those obtained using the split-step Fourier method.

\section{Prestack Migrations of the Marmousi Dataset}

The Marmousi velocity model (Fig. la) contains complex structures with several faults in the upper portion of the model and anticlinal structures in the imaging target region that represents a reservoir (Bourgeois et al., 1991). The Marmousi common-shot-gather dataset was generated by a finite-difference scheme and has become an industry standard dataset to test the capability of different migration algorithns. The Marmousi model was discretized with a horizontal grid spacing of $25 \mathrm{~m}$ and a vertical grid spacing of $4 \mathrm{~m}$. The dataset consists of 240 shots with 96 traces per shot. The shot and receiver intervals are both $25 \mathrm{~m}$. The first shot was at $x=3000 \mathrm{~m}$. Receivers were on the left hand side of each shot with a minimum offset of $200 \mathrm{~m}$. The frequency range used during migrations is from 5 to $60 \mathrm{~Hz}$ with 226 frequency components. Migration images obtained using the split-step Fourier (SSF), extended local Born Fourier (ELBF), extended local Rytov Fourier
(ELRF), quasi-Born Fourier (QBF), and globally optimized Fourier finite-difference (GOFFD) methods are displayed in Figs. 1(b)-(f), respectively.

The structure of the Marmousi velocity model is complex, but the lateral velocity variations are moderate in most regions, therefore, the SSF migration provides a reasonably good image (Figs.lb). Figs.1(c)-(f) show smoother images of the anticlinal structure (labeled "Target Region" in the figures) than Fig.l(b). Several interfaces merge into the region around $\mathrm{A}$ in $\mathrm{Fig.l}$ (a) and hence the wave phenomena in this region are complex. The image around location $A$ in Fig. I (b) obtained using the SSF method is different from that obtained using a time-consuming but more accurate method, the finite-difference wave-equation migration algorithm provided by Advance Geophysical Co. (Bevc, 1997). This is because the split-step Fourier method uses a smal]mangle approximation of the extended local-Rytov Fourier method and accounts only for the phase changes but not for the amplitude changes of wavefields caused by lateral velocity variations. Taking the amplitudes of scattering into account is vital to accurately inaging complex structures such as the area near location A. All of the images in Figs. 1(c)-(f) have similar quality and are comparable to the finite-difference migration image (Bevc, 1997).

The ELBF migration took almost the same CPU time as the SSF migration, the QBF migration took approximately 15 per cent more CPU time, and both the ELRF and GOFFD migrations took approximately 50 per cent more CPU time.

\section{Migrations of an Exploding-Reflector Dataset}

The SEG/EAGE salt model is a complex structure containing salt features situated in the US Gulf of Mexico (Aminzadeh et al., 1996; O'Brien and Gray, 1996). The model contains faults with various dips and strong lateral velocity variations in most regions. This model is not as complex as the Marmousi model, but its lateral velocity variations are stronger than those in the Marmousi model. The velocity contrast between the salt body and the sumrounding sediments is often larger than a factor of two. We chose a 2D slice of the SEG/EAGE 3D salt model (Fig.2a) that was discretized with a grid spacing of $12.192 \mathrm{~m}$. We generated an exploding-reflector dataset using a finite-difference scheme with a fourth-order accuracy in space and a second-order accuracy in time. The wavefield was recorded at the upper boundary of the model. Migrations were carried out using the SSF, ELBF, QBF, ELRF, and GOFFD methods (Figs.2b-f) for a frequency range of $1-40 \mathrm{~Hz}$. The correct locations of salt interfaces are superimposed onto the images with solid gray lines (red lines in color) in Figs.2(b)-(f). The ELBF, QBE, ELRF, and GOFFD migrations give more accurate images near location $A$ (Figs.2c-f) than the SSF migration (Fig,2b). The images obtained from the ELBF and ELRF migrations are similar (Figs.2c, e), while the QBF migration (Fig.2d) and the GOFFD migration (Fig.2f) give more accurate images of the interface at location $B$. In addition, the GOFFD migration (Fig. $2 \mathrm{f}$ ) provides more accurate images of the lower interface of the sait body and those of subsalt interfaces than the other migrations.

Compared to the SSF migration, the QBF migration took approximately 13 per cent more CPU time and the GOFFD migration took approximately 40 per cent more CPU time. The variable extrapolation interval approach (Huang et al., 1998) must be used in the ELBF and ELRF migrations to make them stable for this stat model because it has strong lateral velocity variations, and con- 


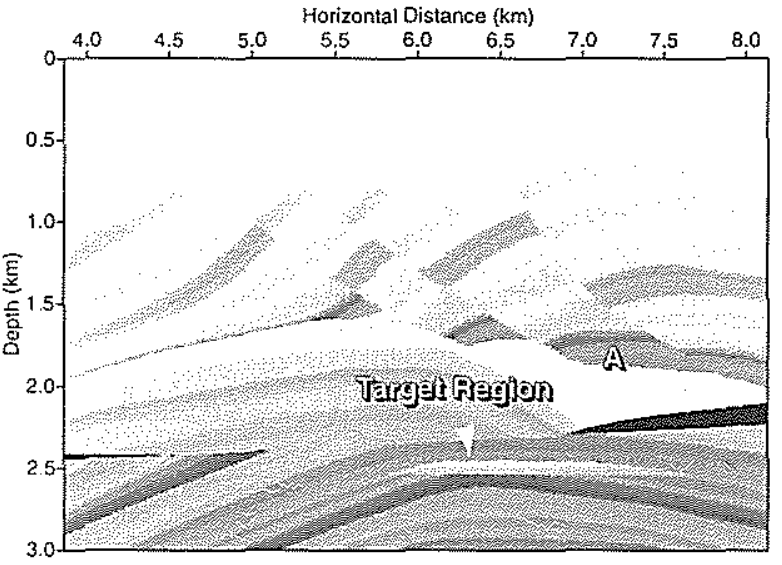

(a) Marmousi velocity model

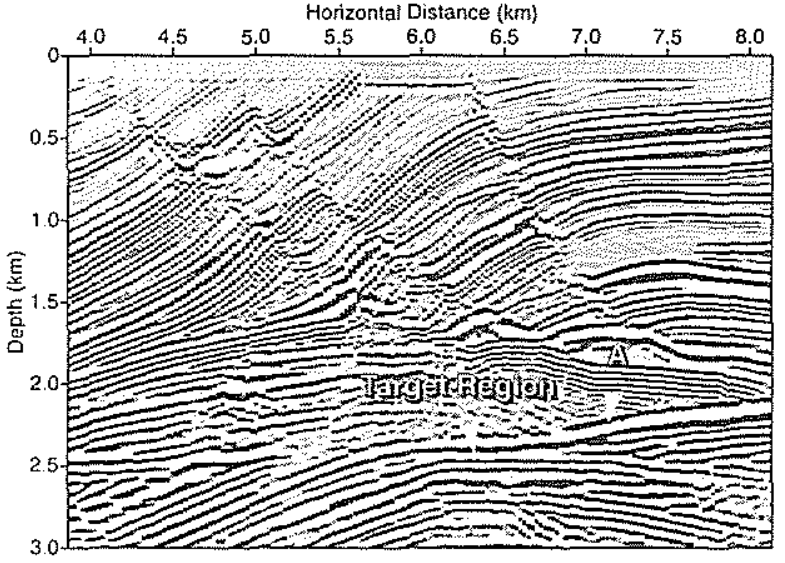

(c) Extended local Born Fourier migration

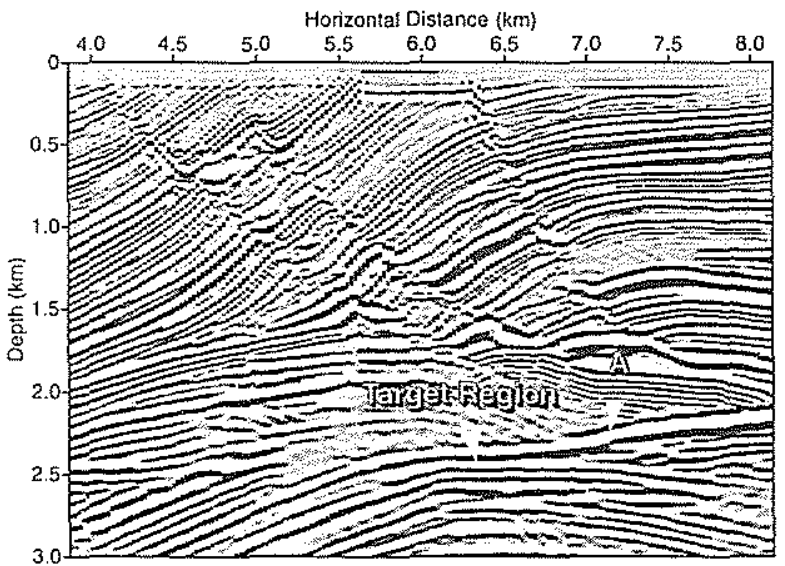

(e) Extended local Rytov Fourier migration

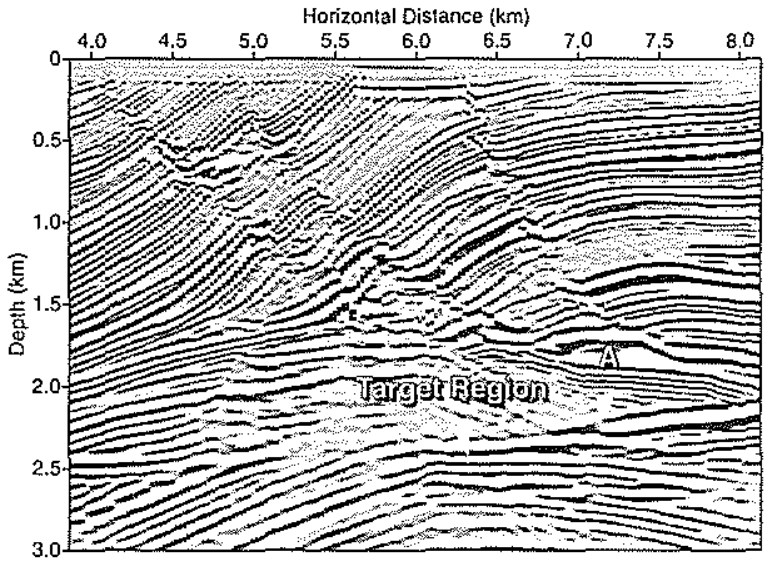

(b) Split-step Fourier migration

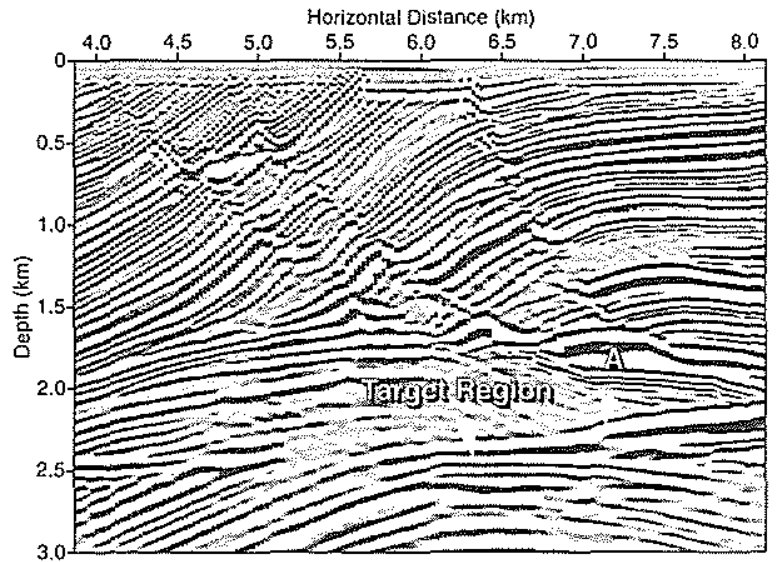

(d) Quasi-Born Fourier migration

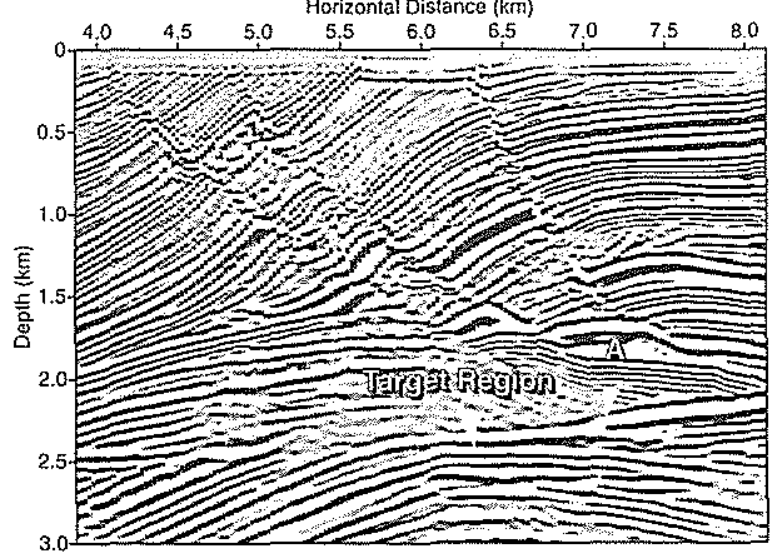

(f) Globally optimized Fourier finite-difference migration

Fig. 1: Marmousi velocity model (a) together with its prestack migration images (b)-(f) obtained using different migration methods. The images in (c)-(l) have a similar quality which is higher than that in (b) (see the target region and location in $A$ in each panel). 


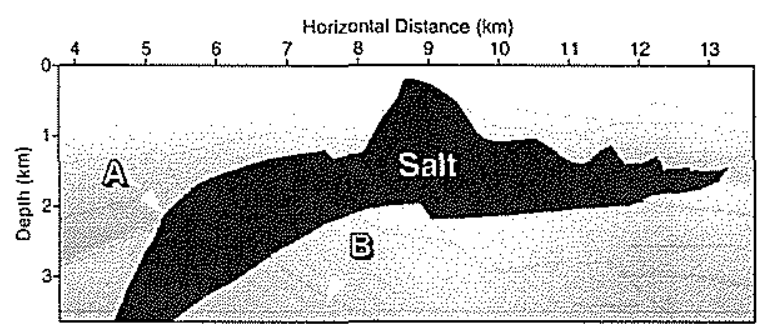

(a) Velocity model

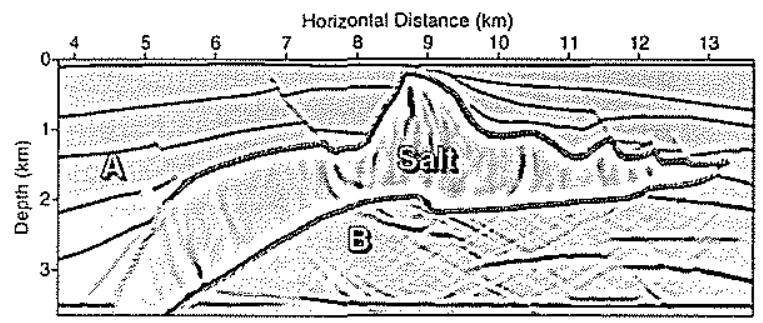

(c) Extended local Born Fourier migration

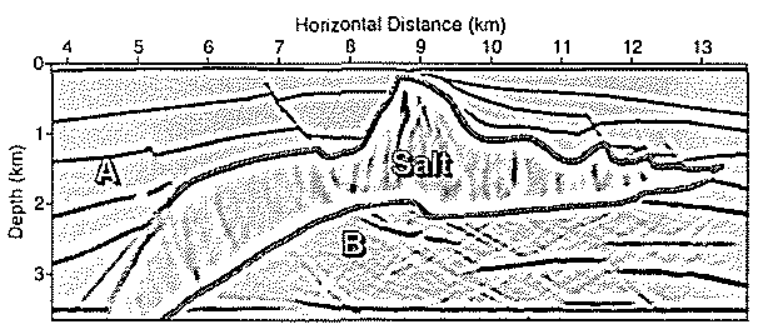

(e) Extended local Rytov Fourier migration

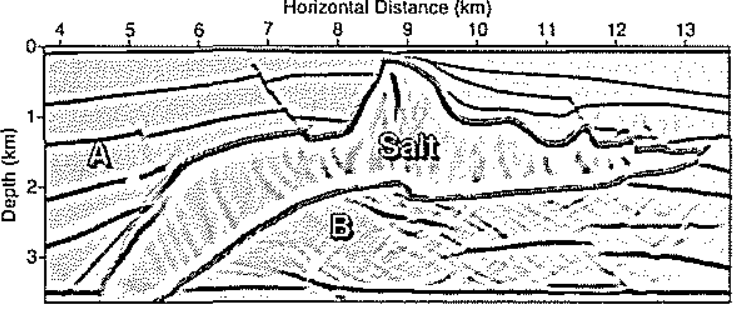

(b) Split-step Fourier migration

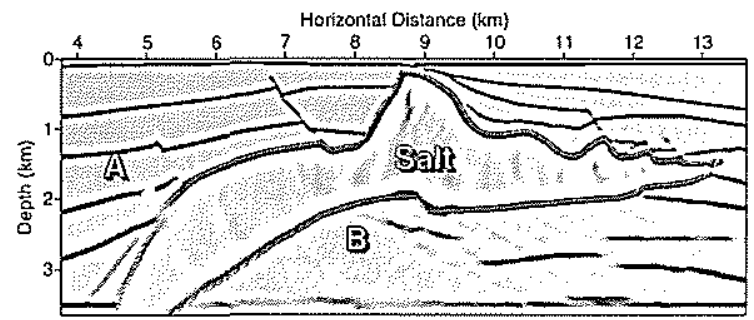

(d) Quasi-Born Fourier migration

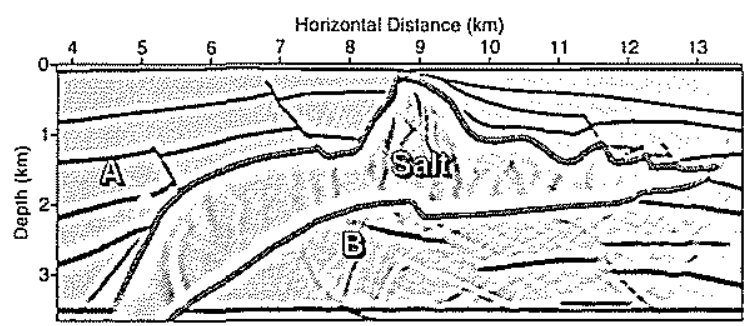

(f) Globally optimized Fourier finite-difference migration

Fig. 2: Velocity model (a) of a 2D slice of the SEG/EAGE model along with its poststack migration images (b)-(f) obtained using different migration methods. The images in (c)-(e) have a similar quality which is higher than that in (b). The image quality in (f) is the best among those shown. The solid gray lines (red lines in color) are the correct locations of the boundaries of the salt body.

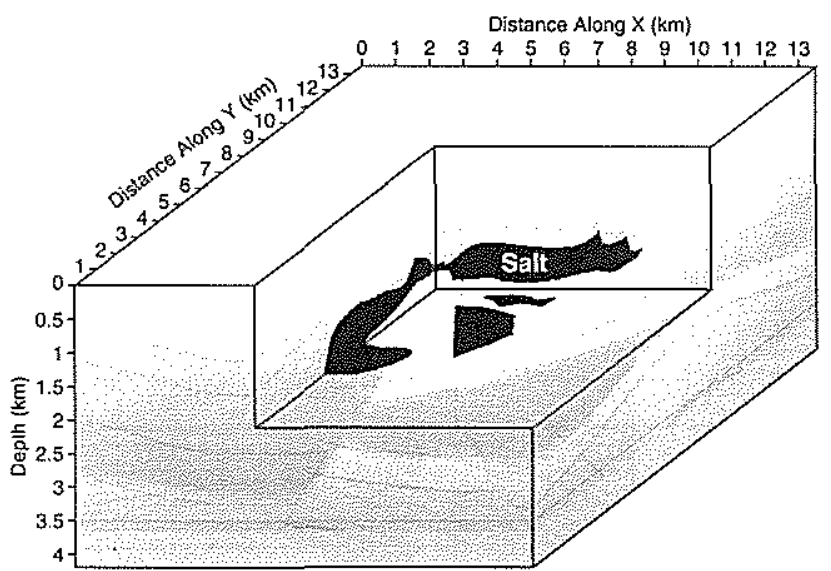

(a) 3D velocity model

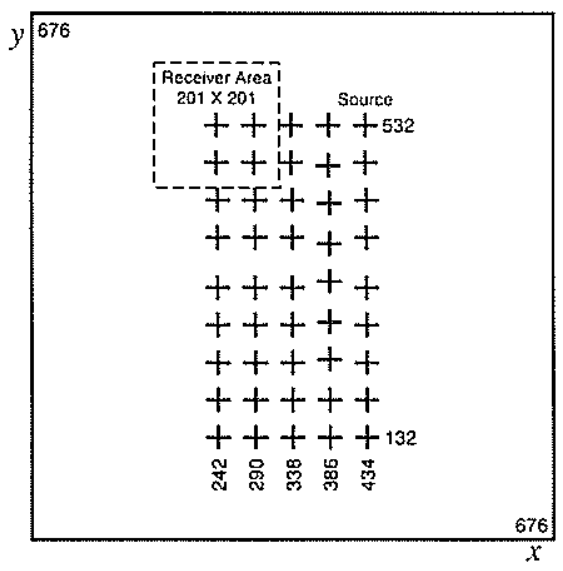

(b) Source location

Fig. 3: Velocity model (a) of the SEG/EAGE 3D model together with the source locations at the top the model (b). 


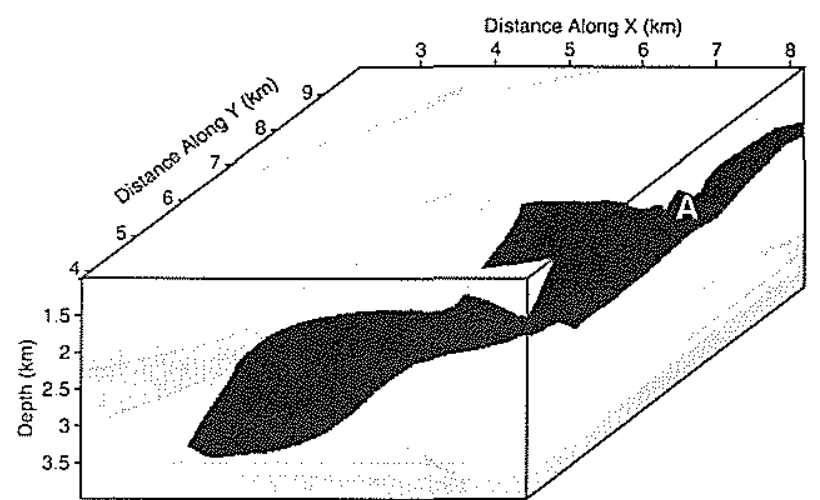

(a1) Front, right and top sides of a $3 \mathrm{D}$ velocity cube

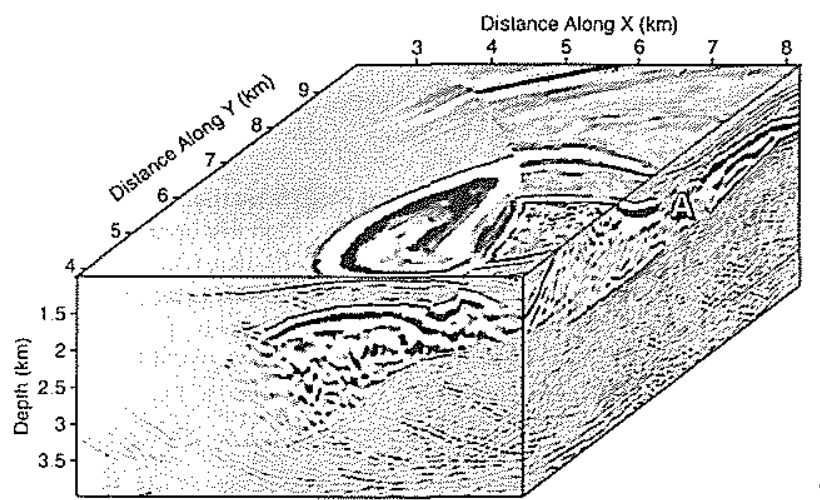

(a2) Split-step Fourier migration

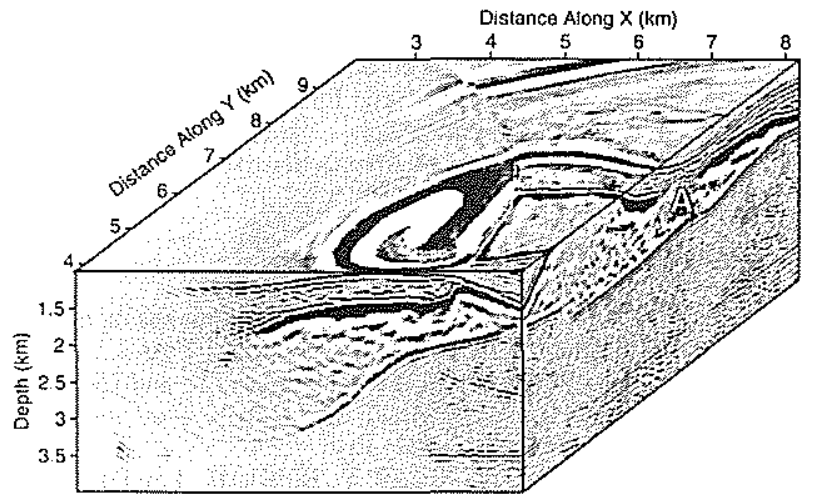

(a3) Globally optimized Fourier finite-difference migration

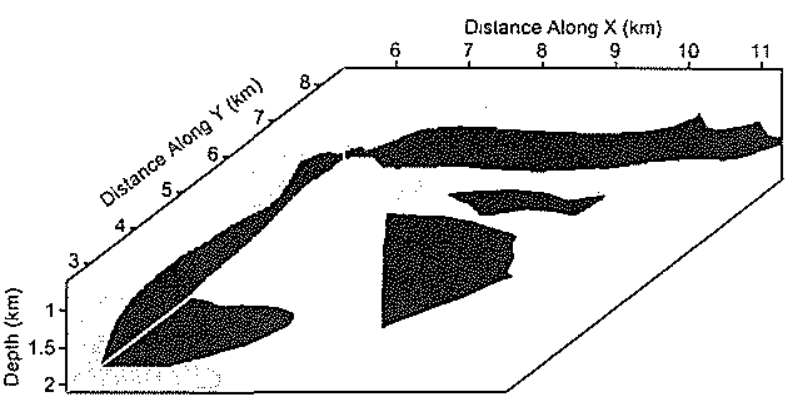

(bl) Back, left and bottom sides of a 3D velocity cube

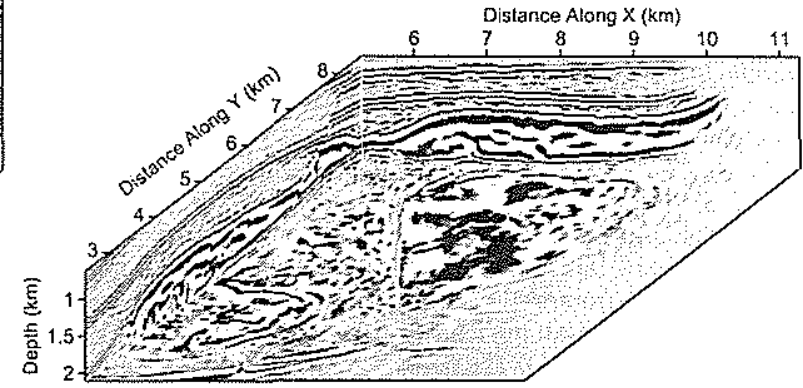

(b2) Split-step Fourier migration

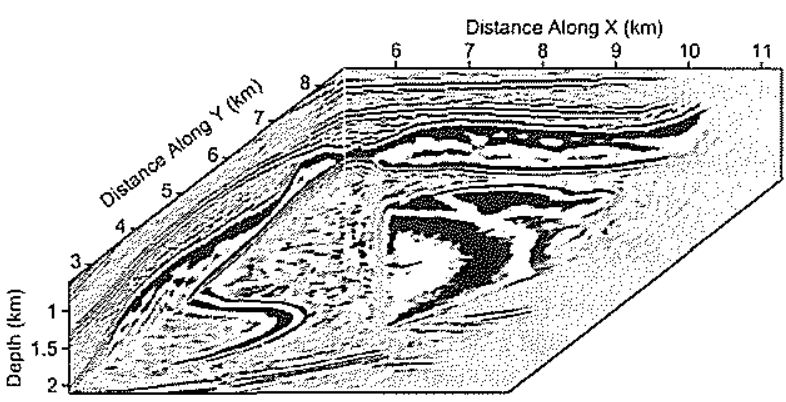

(b3) Globally optimized Fourier finite-difference migration

Fig. 4: Velocity models (al and b1) of two sub-cubes of the SEG/EAGE 3D salt model together with their migration images obtained using the split-step Fourier method (a2 and b2) and the globally optimized Fourier finite-difference method (a3 and b3). The image quality in (a3) and (b3) is much better than that in (a2) and (b2).

sequently, the ELBF and ELRF migrations took, respectively, approximately 1.5 times and 1 time more CPU time than the SSF migration.

\section{D Prestack Migrations of a Common-Shot Dataset}

3D prestack migrations were performed on a dataset consisting of 45 common-shot gathers (Ober et al., 1997) extracted from a synthetic dataset that was generated using a finite-difference scheme 


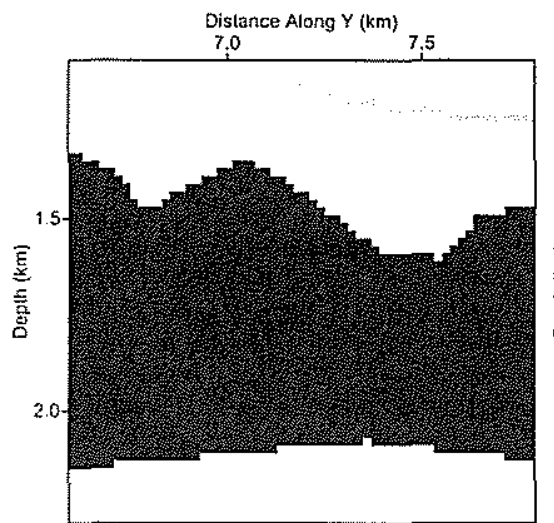

(a) Velocity model

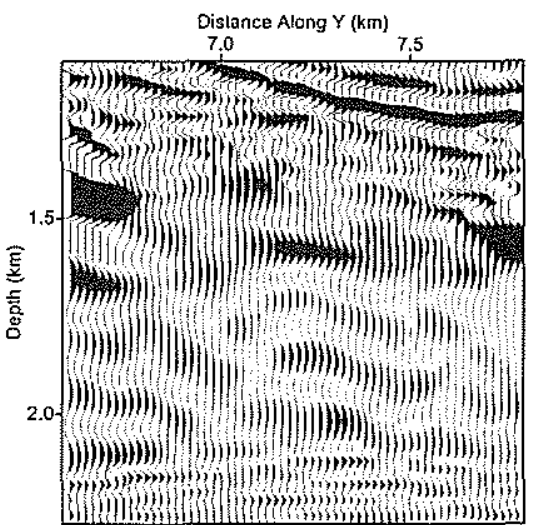

(b) Split-step Fourier migration

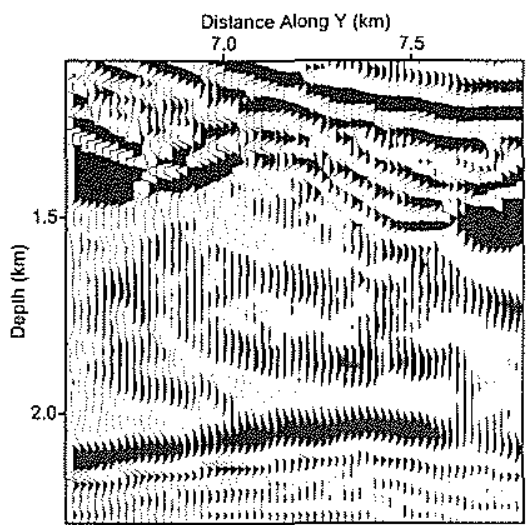

(c) Globally optimized Fourier FD migration

Fig. 5: Velocity model (a) around location A in Fig.4 together with images obtained using the split-step Fourier method (b) and the globally optimized Fourier finite-difference method (c). The upper and lower boundaries of the salt body are well imaged in (d) but not in (c).

for the SEG/EAGE 3D salt model (Fig.3a). The model was defined on a 3D grid with grid spacings of $20 \mathrm{~m}$ along the $x-, y$-, and $z$ directions. The sources were located at the top of the model and distributed as shown in Fig.3b. Each common-shot gather consists of $201 \times 201$ traces at receivers centered around the source location. Migrations were made using the SSF and GOFFD methods for a frequency range of $2-30 \mathrm{~Hz}$. The panels on the left side of Fig. 4 show the front, right and top sides of a 3D velocity subcube of the SEG/EAGE salt model (Fig. $4 \mathrm{al}$ ) and the images of the subcube (Figs. $4 \mathrm{a}_{2}$ and $\mathrm{a} 3$ ). The panels on the right side of Fig. 4 show the back, left and bottom sides of another 3D velocity subcube of the SEG/EAGE salt model (Fig. $4 \mathrm{bl}$ ) and the images of the subcube (Figs. 4 b2 and b3). The GOFFD migration (Figs. $4 \mathrm{a} 3$ and b3) produces images with a significantly improved quality compared to that of the SSF migration images (Figs. $4 \mathrm{a} 2$ and $\mathrm{b} 2$ ). For detailed comparison, the images around the areas near location A in Fig. 4 a2 and Fig. 4 a 3 are shown in Fig.5 where Fig.5a is the velocity model in that area.

The GOFFD migration took approximately 30 per cent more computational time than the SSF migration.

\section{CONCLUSIONS}

The extended local Born Fourier, quasi-Born Fourier, extended local Rytov Fourier, and globally optimized Fourier finite-difference methods are all more accurate than the split-step Fourier scheme. The former three Fourier transform-based methods are more suitable for imaging complex structures having moderate lateral velocity variations because they are all based on first-order approxintations. When lateral velocity variations are moderate such that no variable extrapolation intervals are needed in the extended local Born Fourier method, the method is as efficient as the splitstep Fourier scheme. The quasi-Born Fourier method is approximately 15 per cent more computationally expensive than the splitstep Fourier method, and both the extended local Rytov Fourier and globally optimized Fourier methods are approximately 4050 per cent more expensive. Because the extended local Born Fourier and quasi-Born Fourier methods account for amplitudes of wavefields more accurately than the other methods, they could be used for true-amplitude migration. The globally optimized Fourier finite-difference approach - a hybrid split-step Fourier and finitedifference method - is an accurate and efficient tool for imaging structures having strong lateral velocity variations. For 3D prestack migration, the method is approximately 30 per cent more computationally expensive than the split-step Fourier scheme.

\section{ACKNOWLEDGMENTS}

We thank Chuck C. Burch and Curtis C. Ober for providing us the 45 common-shot gathers of the synthetic dataset for the SEG/EAGE 3D salt model. This work was funded by the US Department of Energy Office of Basic Energy Sciences through contract W-7405-ENG-36 to Los Alamos National Laboratory. This is contribution No. 20 of the Los Alamos Seismic Research Center:

\section{REFERENCES}

Abma, R., Sun, J., and Bernitsas, N., 1999, Antialiasing methods in kirchhoff migration, Geophysics, 64, 1783-1792.

Aminzadeh, F., Burkhard, N., Long, J., Kunz, T., and Duclos, P., 1996, Three dimensional SEG/EAEG models - an update, The Leading Edge, 15, No. 2, 131-134.

Audebert, F., Nichols, D., Rekdal, T., Biondi, B., Lumley, D., and Urdaneta, H., 1997, Imaging complex geologic structure with single-arrival Kirchhoff prestack depth migration, Geophysics, $62,1533-1543$

Bevc, D., 1997, Imaging complex structures with semirecursive Kirchhoff migration, Geophysics, 62, 577-588.

Bourgeois, A., Bourget, M., Lailly, P., Poulet, M., Ricarte, P., and Versteeg, R., 1991, Marmousi, model and data, Eur: Asson. Expl. Geophys., The Marmousi Experience, Versteeg, R., and Grau, G., Eds., Houten, the Netherlands, 5-16.

Claerbout, J. F., 1985, Imaging the Earth's Interior, Blackwell Scientific Publications, Oxford.

de Hoop, M. V., Wu, R., and Le Rousseau, J. H., 2000, General for- 
mulation of screen methods for the scattering of acoustic waves, Wave Motion, 31, 43-70.

Dellinger, J. A., Gray, S. H., Murphy, G. E., and Etgen, J. T., 2000, Efficient 2.5-D true-amplitude migration, Geophysics, 65, 943950.

Fehler, M. C., and Huang, L., 1998, Using local Born and local Rytov Fourier modeling and migration methods for investigation of heterogeneous structures, The 4th SEGJ Intern. Symp. - Imaging Technology, Soc. Expl. Geophys. Japan, Expanded Abstracts, $7-10$.

Fehler, M., Sato, H., and Huang, L., 2000, Envelope broadening of outgoing waves in 2D random media: A comparison between the Markoc approximation and numerical simulations, Bull. Seis. Soc. Am. 90, 914-928.

Fei, T., Fehler, M. C., and Hildebrand, S. T., 1996, Depth migration artifacts associated with first-arrival traveltime, $66 \mathrm{th} \mathrm{Ann}$. Internat. Mtg., Soc. Expl. Geophys., Expanded Abstracts, 499--502.

Gazdag, J., and Sguazzero, P., 1984, Migration of seismic data by phase-shift plus interpolation, Geophysics, 49, 124-131.

Gazdag, J., 1978, Wave equation migration with the phase-shift method, Geophysics, 43, 1342-1351.

Geoltrain, S., and Brac, J., 1993, Can we image complex structure with lst-arrival travel-time?, Geophysics, 58, 564-575.

Gray, S. H., and May, W. P., 1994, Kirchhoff migration using eikonal equation travel-times, Geophysics, 59, 810-817.

Hale, D., 1991, 3-D depth migration via McClellan transformations, Geophysics, 56, 1778-1785.

Hanitzsch, C., 1997, Comparison of weights in prestack amplitudepreserving Kirchhoff depth migration, Geophysics, 62, 18121816.

Hildebrand, S. T, and Fehler, M. C., 1998, Kirchhoff-Born migration, 68th Ann. Internat. Mrg., Soc. Expl. Geophys., Expanded Abstract, 1531-1533.

Hokstad, K., 2000, Multicomponent Kirchhoff migration, Geophysics, $65,861-873$.

Huang, L., and Fehler, M. C., 1998a, Accuracy analysis of the splitstep Fourier propagator: implications for seismic modeling and migration, Bull. Seis. Soc: Am., 88, 18-29.

Huang, L., and Fehler, M. C. 1998b, Extended local Rytov Fourier method for fast simulation of primary forward acoustic wave propagation in a heterogeneous medium, 1998 Fall Meeting, American Geophysical Union, EOS, Transactions, vol.78, No.46, pF425.

Huang, L., and Fehler, M. C., 2000a, Globally optimized Fourier finite-difference migration method, 70th Ann. Internat. Mtg., Soc: Expl. Geophys, Expanded Abstracts, 802-805.

Huang, L., and Fehler, M. C. 2000b, Quasi-Born Fourier migration, Geophys. J. Intern., 140, 521-534.

Huang, L., and Fehler, M. C., 2001, Globally optimized Fourier finite-difference migration in three dimensions, The 5th SEGJ Intern. Symp. - Imaging Technology, Soc. Expl. Geophys. Japan, Expanded Abstracts.

Huang, L., and Wu, R., 1996a, 3-D prestack depth migration with an acoustic pseudo-screen propagator, in Hassanzadeh, S., Ed., Mathematical Methods in Geophysical Imaging IV, Proc. SPIE: The International Society for Optical Engineering, 2282, 40-51.

Huang, L., and Wu, R. 1996b, Prestack depth migration with acoustic screen propagators, 66th Ann. Internat. Mtg., Soc. Expl. Geophys., Expanded Abstracts, 415-418.

Huang, L., Fehler, M. C., and Burch, C. C., 1998, A hybrid local
Born/Rytov Fourier migration method,, in Hassanzadeh, S., Ed., Mathematical Methods in Geophysical Imaging V, Proc. SPIE: The International Society for Optical Engineering, 3453, 14-25.

Huang, L., Fehler, M. C., Roberts, P. M., and Burch, C. C., 1999a, Extended local Rytov Fourier migration method, Geophysics, 64, 1535-1545.

Huang, L., Fehler, M. C., and Wu, R. 1999b, Extended local Born Fourier migration method, Geophysics, 64, 1524-1534.

Jin, S., and Wu, R., 1999, Depth migration with a windowed screen propagator, J. of Seismic Expl., 8, 27-38.

Keho, T. H., and Beydoun, W., 1988, Paraxial ray Kirchhoff migration, Geophysics, 53, 1540-1546.

Le Rousseau, J. H., and De Hoop, M. V., 1999, Modeling and imaging with scalar generalized-screen algorithms in isotropic media, Geophysics, Submitted.

Moser, T. J., 1994, Migration using the shortest-path method, Geophysics, 59,1110-1120.

Ober, C. C., Oldfield, R. A., Womble, D. E., and Mosher, C. C., 1997, Seismic imaging on massively parallel computers, $67 \mathrm{~h}$ Ann. Internat. Mtg., Soc. Expl. Geophys., Expanded Abstracts, $1418-1421$

O'Brien, M. J., and Gray, A. H., 1996, Can we image beneath salt?, The Leading Edge, 15, No. 1, 17-22.

Ristow, D, and Rühl, T., 1994, Fourier finite-difference migration, Geophysics, 59, 1882-1893.

Romero, L. A., Ghiglia, D. C., Ober, C. C., and Morton, S. A., 2000 . Phase encoding of shot records in prestack nigration, Geophysics, 65, 426-436.

Schneider, W. A., 1978, Integral formulation for migration in 2 and 3 dimensions, Geophysics, 43, 49-76.

Stoffa, P. L., Fokkema, J. T., de Luna Freire, R. M., and Kessinger, W. P., 1990, Split-step Fourier migration, Geophysics, 55, 410421.

Stolt, R. H., 1978, Migration by Fourier transform, Geophysics, 43, $23-48$.

Sun, Y., Qin, F., Checkles, S., and Leveille, J. P., 2000, 3-D prestack Kirchhoff beam migration for depth imaging, Geophysics, 65 . $1592-1603$.

Tygel, M., Schleicher, J., Hubral, P., and Santos, L. T., 1998, 2.5D true-amplitude Kirchhoff migration to zero offset in lateraliy inhomogeneous media, Geophysics, 63, 557--573.

Wiggins, J. W., 1984, Kirchhoff integral extrapolation and migration of nonplanar data, Geophysics, 49, 1239-1248.

Wu, R., and Jin, S., 1997, Windowed GSP (generalized screen propagators) migration applied to SEG-EAEG salt model data 67th Ann. Internat. Mtg. Soc. Expl. Geophys., Expanded Abstracts, 1746-1749.

Xie, X., and Wu, R., 1998, Improve the wide angle accuracy of screen method under large contrast, 68th Ann. Internat. Mtg.. Soc. Expl. Geophys., Expanded Abstracts, $1811-1814$.

Zhu, T., 1988, Ray-Kirchhoff migration in inhomogeneous-media, Geophysics, 53, 760-768. 